\title{
A commentary on "Benefit of a nurse-led telephone-based intervention prior to the first urogynecology outpatient visit: a randomized-controlled trial"
}

\author{
Dominique Malacarne Pape ${ }^{1}$ \\ Received: 20 April 2020 / Accepted: 28 April 2020 / Published online: 15 May 2020 \\ (C) The International Urogynecological Association 2020
}

This was a randomized-controlled trial conducted at a large academic tertiary referral center. The purpose of the research was to gain information regarding the usefulness of nurse-led telephone preparation visits as a way to reduce workload and increase efficiency during in-person medical consultation, in an era where clinics are becoming overcrowded and resource constraints are on the rise. The aim of this trial was to evaluate whether or not a pre-visit telephone interview would be associated with the reduction in first urogynecology visit duration and improved patient and physician satisfaction.

One hundred eight patients scheduled for their very first urogynecological visit were recruited, of which 101 were evaluable, 53 allocated to the intervention group and 48 in the control group. The intervention consisted of a telephone interview which included questions about current complaints and medical history. The patient was asked to complete a voiding diary to bring with her to the visit, as well as prior medical records. Patient satisfaction with the clinic visit was measured using the ZUF-8 questionnaire, and physician satisfaction was evaluated using standardized questions including one about the estimated time economy of the visit. The two groups did not differ demographically. The mean visit duration in the intervention group was 26 min (SD 8.9) versus $30.8 \mathrm{~min}$ (SD 11.4) in the control group, with a significant difference of $4 \min 50 \mathrm{~s}(p=0.02)$. There was no difference in mean ZUF-8 score between groups, and both groups reported a high proportion of "excellent" quality of consultation (78\% intervention, $71.1 \%$ control). Additionally, $82.69 \%$ of physicians responded that they were satisfied with patient preparation in the intervention group.

In summary, the researchers identified a time-saving effect of nearly $5 \mathrm{~min}$ per patient in the intervention group, which they extrapolated to correlate with one additional patient seen per half clinic day. This consultation time difference did depend on diagnosis, with urinary incontinence taking the longest, indicating this intervention may be more beneficial for certain groups of patients. Both patients and physicians were satisfied with the intervention. Study of the long-term impact on the costeffectiveness of this intervention is warranted, as preemptive telephone consultation in certain domains of urogynecology may improve efficiency during the initial clinic visit and decrease the overall number of follow-up visits and costs per patient.

Dominique Malacarne Pape

Dominique.Malacarne@nyulangone.org

1 NYU Langone Health, New York, NY, USA 